\title{
New promising potential in fighting atherosclerosis: HDL and reverse cholesterol transport
}

\author{
Ginter $\mathrm{E}^{1}$, Simko $\mathrm{V}^{2}$ \\ Institute of Preventive and Clinical Medicine, Bratislava, Slovakia. ginter.emil@mail.t-com.sk
}

\begin{abstract}
Atherogenesis and cardiovascular disease (CVD) mortality remain an outstanding population health risk. Its importance grows with rising affluence leading to obesity in the developing countries. Inspite of an enormous initial enthusiasm related to the breakthrough success with the statins, there remains a substantial residual risk of CVD. While statins are effective in lowering the low density lipoproteins, LDL (the „bad“ cholesterol), the residual risk is also related to unsatisfactorily low levels of high density lipoprotein, HDL (the "good“ cholesterol). It has been long known that low levels of HDL are associated with CVD as an independent risk factor. The original concept of a direct transfer of cholesterol from LDL to HDL has been proved to be oversimplified. Recent studies confirm direct participation of HDL in anti-atherogenesis by promoting the efflux of cholesterol contained in the foam cells that constitute atherosclerotic lesions. The challenge is to identify therapeutic interventions aimed at raising HDL, thus assisting statins that have until recently been mostly used as a monotherapy (Fig. 5, Ref. 26). Full Text in PDF www.elis.sk.

Key words: low-density lipoprotein cholesterol (LDL), high-density lipoprotein cholesterol (HDL), reverse cholesterol transport, apoprotein apo A-I, apoprotein apo A-II, foam cells, anti-atherogenic action.
\end{abstract}

CVD remains a major cause of worldwide morbidity and mortality despite therapeutic advances that control many risk factors such as the LDL to attain levels lower than previously possible. Several large statin trials and meta-analyses have demonstrated a reduction in LDL and CVD morbidity and mortality (1). This promoted optimistic predictions that the CVD in the near future will cease to be the predominant cause of population mortality. In reality, some West European countries (e.g France) distinguished themselves by a prominent drop in CVD mortality, CVD becoming a lower threat than neoplasms. Yet, in Central Europe and mainly in the European East, CVD reamains the most prominent health threat, the main cause of mortality (2) (Fig. 1).

\section{Atherosclerosis: The present concept}

Existing consensus on the pathogenesis of atherosclerosis is the result of advances in understanding of the lipoprotein metabolism in the 1950s and in 1960s, and also in achievements of modern cellular biology in the 1970s (3-5).

Atherosclerosis is associated with increased endothelial permeability of monocytes under the influence of adhesion molecules (e.g. selectins E and P). This promotes endocytosis of oxidized LDL (monocyte chemotactic protein-1, MCP-1) which carries serum cholesterol. Circulating LDL particles enter the arterial wall

${ }^{1}$ Institute of Preventive and Clinical Medicine, Bratislava, Slovakia, and ${ }^{2}$ State University of New York, Downstate Medical Center at Brooklyn, USA

Address for correspondence: E. Ginter, RND, DSc, Racianska 17, SK83102 Bratislava, Slovakia.

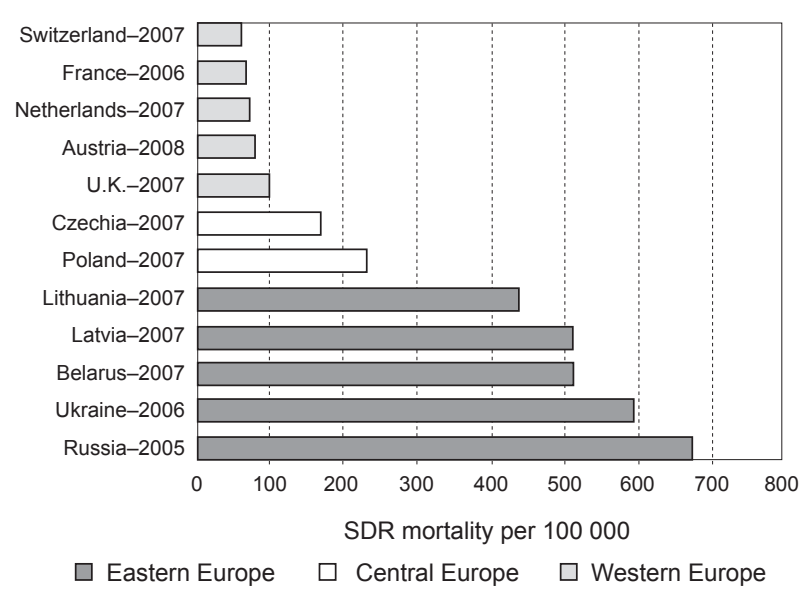

Fig. 1. CVD mortality in Europe. According to WHO (2).

and accumulate in the intima, where they undergo further oxidation and promote plaque formation. Intimal macrophages internalize oxidized LDL particles through scavenger receptors and become foam cells - a key process in the development of atherosclerotic plaque (Fig. 2).

Further progression, an increase in macrophages and foam cells, promotes the plaque instability. The arterial lumen is tenuously protected by an extracellular matrix -fibrous cap- that separates the atherosclerotic plaque from the vessel lumen. This protective layer tends to be weak and prone to disruption. Thrombogenic material of the plaque becomes easily exposed to the circulating blood and it triggers a thrombus formation with partial or complete arterial occlusion. 


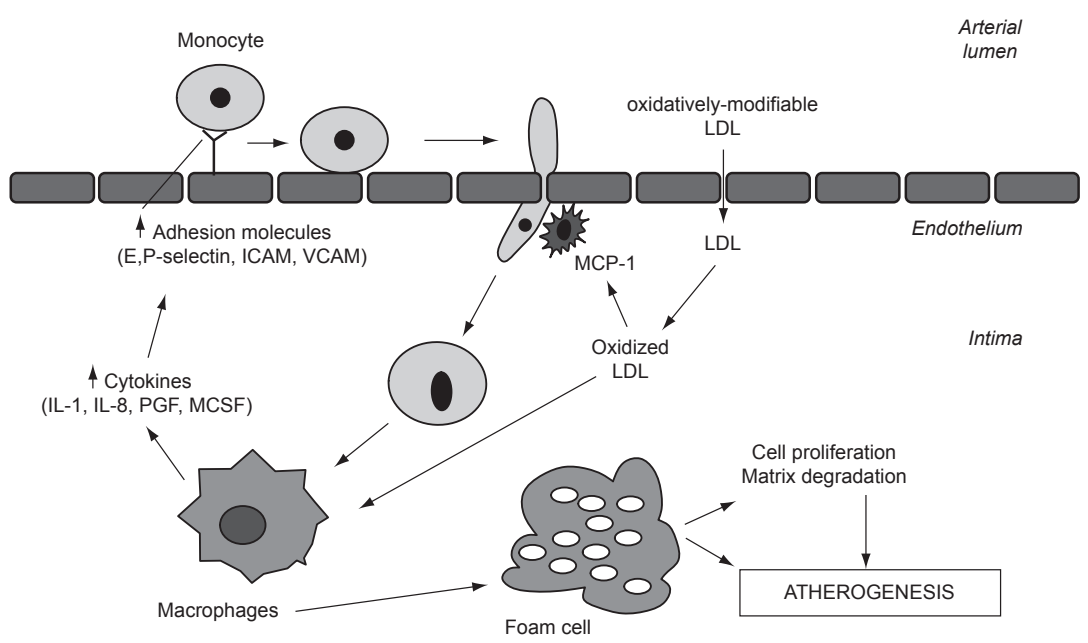

Fig. 2. Mechanism of atherogenesis. Partly according to Rocha and Libby (5).

A sobering fact is that the statins which primarily lower LDLcholesterol, only reduce the risk of atherosclerosis by $30 \%$. Thus $70 \%$ of the risk of atherogenesis still has to be explained and avoided. New data on the metabolism of HDL appear promising at filling at least a part of this residual risk.

\section{HDL structure, metabolism and reverse cholesterol transport}

Our knowledge of HDL dates back to 1929 when Macheboeuf at the Pasteur Institute in Paris isolated a lipoprotein, later recognized as HDL. Then in early 1950s, it was discovered that patients with CVD had low levels of HDL. This lipoprotein abnormality was recognized as a significant and an independent risk factor for CVD (6-12).

Initially, the beneficial influence of HDL was attributed to modifying the LDL, lowering their level directly in the plasma. Now it is recognized that the HDL metabolism is more complex. A nascent flat form of cholesterol-free HDL originates in the liver and in the intestine. The nascent HDL particles, consisting of phospholipid and apolipoprotein A-I, interact with peripheral cells and increase in size as they circulate through the bloodstream (Fig. 3): $\mathrm{HDL}_{2 \mathbf{b}}, \mathrm{HDL}_{2 \mathbf{a}}, \mathrm{HDL}_{3 \mathbf{a}}, \mathrm{HDL}_{3 \mathbf{b}}$ and $\mathrm{HDL}_{3 \mathbf{c}}$.

Further metabolism of HDL particles is complex (Fig. 4). HDLs are capable of picking up cholesterol, from macrophages and other cells by interaction with the ATP-binding cassette transporters ABC. HDLs are then converted into mature cholesterol ester-rich HDLs as a result of the plasma cholesterol-esterifying enzyme lecithin-cholesterol acyltransferase (LCAT). In humans, probably the most relevant pathway of HDL catabolism is mediated by cholesteryl ester transfer protein (CETP). CETP promotes the transfer of cholesteryl esters from antiatherogenic HDLs to apolipoprotein B containing lipoproteins LDL, which are quickly taken up by apo B receptors in the liver.

The so-called "reverse cholesterol transport pathway" is believed to be the main protective effect of $\operatorname{HDL}(10,11,14)$. This pathway results in the transfer of excess cholesterol from peripheral cells - such as macrophages in atherosclerotic plaques, to HDL, which transfers its cholesterol to the liver for excretion in the form of bile acids or free cholesterol. The concentration of large high density lipoprotein particles more accurately reflect protective
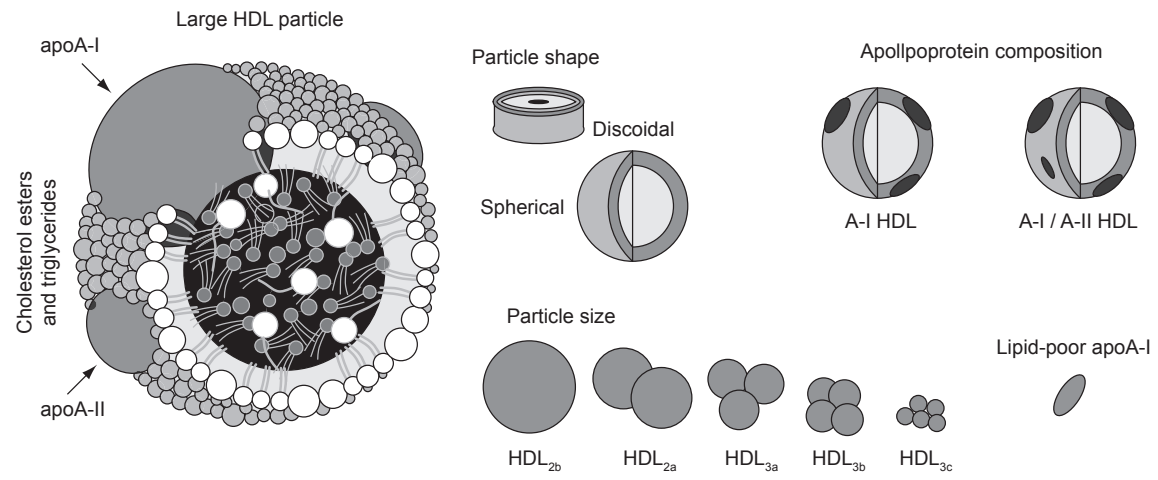

Fig. 3. Anatomy of HDL structure and subpopulations of HDL particles. Partly according to EHJ Supplement (Eur Soc Cardiol 2011). 


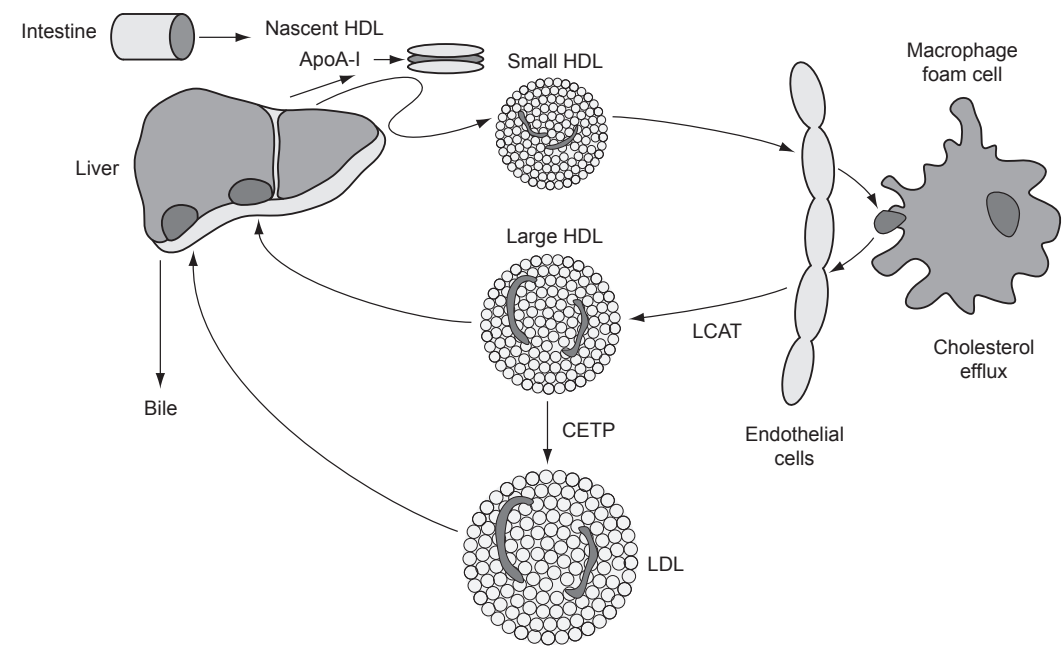

Fig. 4. The sheme of the reverse cholesterol transport pathway. Eplanation to figure: Scavenger receptor class B member 1 (SRB1) also known as SR-BI is a protein that in humans is encoded by the SCARB1 gene. SR-BI functions as a receptor for high-density lipoprotein. LDL-R specific receptor for LDL; ABC - ATP-binding cassette transporters of cholesterol; CETP - cholesteryl ester transfer protein, Partly according to Remaley and Warnick (12).

action, as opposed to the concentration of total high density lipoproteins particles. A very large number of genetic polymorphisms influence the serum level of HDL particles in any given individual.

Regarding HDL catabolism in man, probably the most relevant pathway is mediated by cholesteryl ester transfer protein (CETP). CETP promotes the transfer of cholesteryl esters from antiatherogenic HDL to apolipoprotein B containing lipoproteins, predominantly the LDL. These are promptly taken up by the apo B liver receptors. Animals that lack CETP, such as the rat and dog, have very high HDL. Other species that have large amounts of CETP, such as rabbits, monkeys and humans, have low HDL levels (13). Therefore, inhibiting CETP appears to be an effective means to raise HDL. However, the metabolism of HDL is complex. A very large number of genetic polymorphisms influences the serum level of HDL in any given individual.

The main protective effect of HDL is presumably related to a „reverse cholesterol transport pathway“. By this mechanism excess cholesterol is transferred from peripheral cells - for instance from macrophages in atherosclerotic plaques - to the HDL that eventually carries its cholesterol to the liver for excretion in the form of bile acids or free cholesterol.

\section{HDL, cholesterol efflux capacity and atherogenesis}

The Framingham Heart Study in the late 1960s revealed that the men and women who had low levels of HDL cholesterol (less than $0.9 \mathrm{mmol} / \mathrm{L}$ ) had eight times the risk of CVD compared to those with HDL-cholesterol levels above $1.7 \mathrm{mmol} / \mathrm{L}$. Low levels of HDL were later confirmed in a large number of clinical and epidemiological studies to be strongly associated with an increase in the risk of CVD.

The concept of a "reverse cholesterol transport pathway" is now considered to be the essential protective effect of $\operatorname{HDL}(10,11,14)$. HDL circulates in various forms, including both lipid-rich and

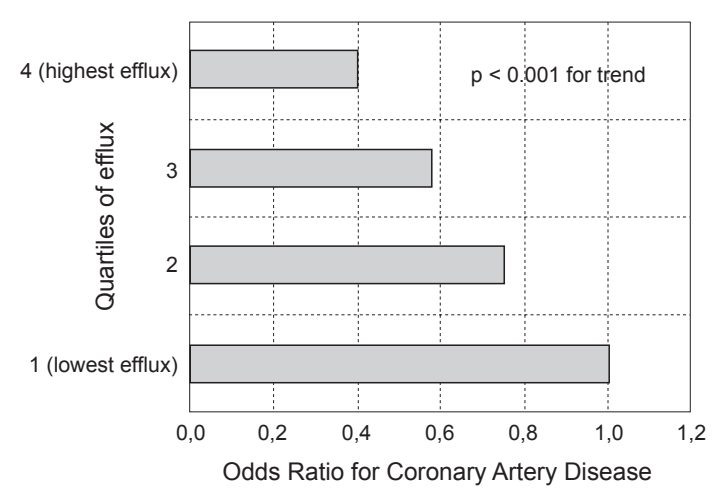

Fig. 5. Coronary artery disease status according to quartiles of cholesterol efflux capacity from macrophages. According to the data of Khera et al (11).

lipid-poor subfractions. The lipid-poor preß1-HDL fractions acquire cholesterol from macrophages in atherosclerotic plaques. In this process a prominent transfer role depends on specific proteins, ATP-binding cassette (ABC) transporters. Subsequently, cholesterol acquired by HDL is cleared in the liver for excretion (Fig. 4).

Cholesterol efflux capacity from macrophages, a measure of HDL function, has a strong inverse association with both carotid intima-media thickness and the likelihood of angiographic coronary artery disease. The functionality of HDL for cholesterol transfer, its efflux capacity in an individual has been revealed by using sophisticated techniques. Khera et al (11) used murine macrophages radiolabeled with ${ }^{3} \mathrm{H}$-cholesterol. Efflux mediums containing serum devoid of apoprotein B were then added for 4 hours. Liquid scintillation counting quantified the efflux of ${ }^{3} \mathrm{H}$-cholesterol from the macrophages. The results were compared with the carotid intima-media thickness. Division of the cohort into quartiles according to efflux capacity from macrophages documented an evident inverse association between efflux capacity and CVD status (Fig. 5). 


\section{HDL and oxidative stress}

Adverse factors may impair the beneficial metabolic function of HDL. Oxidative stress and resulting inflammatory processes have prominent role in atherogenesis. This may also affect the oxidation of lipoproteins which becomes an important component in atherosclerosis. Oxidized LDL induces inflammation by promoting adhesion and influx of monocytes into the intima.

Oxidation impairs not only LDL but also HDL, rendering it dysfunctional. In patients with CVD, oxidated HDL becomes ineffective, yielding a pro-inflammatory and pro-oxidative phenotype (15). There is the possibility that HDL carbamylation contributes to foam cell formation in atherosclerotic lesions (16). Dysfunctional, pro-inflammatory HDL might directly influence monocyte gene expression and function (17).

Presently, it is difficult to dissect the complex metabolic relations between HDL and disease states. Multiple clinical studies have identified individuals with a significant atherosclerotic burden despite normal or elevated levels of HDL. Clinical conditions associated with inflammation and oxidative stress also adversely affect the normal functions of HDL. Other disease processes, proinflammatory and proneoplastic, may also depend on metabolism of HDL. Most recently (18) it was reported that high levels of HDL were associated with lower risk of colon cancer.

When considering to modify the HDL level, it is important that increases in HDL up to $40 \%$ were not associated with higher non-CVD death. The increase in adverse CVD events observed in some trials where HDL cholesterol was adequate or substantially elevated may have been related to medications used by patients rather than to HDL cholesterol level (19).

What are the opportunities to directly promote the beneficial role of HDL? Dysfunctional HDL may be returned to normal by diet, exercise, modification in fat intake and by pharmacologic interventions $(20,21)$. Therapeutic strategies have included niacin, fibrates, thiazolidinediones and bile acid sequestrants. Newly developed pharmacological agents include apolipoprotein A-I mimetics and the cholesteryl ester transfer protein (CETP) inhibitors (22). Orally active mimetic proteins are in development and have shown clinical promise.

The long-acting formulations of nicotinic acid remain the most effective and best tolerated pharmacological strategy for raising HDL in patients already on statin therapy to control LDL. Niacin treatment was reported to cause a moderate increase in the ability of HDL to promote net cholesterol efflux (23). Anacetrapib is a cholesterol ester transfer protein inhibitor. Treatment with anacetrapib had robust effects on LDL and HDL and during the study no adverse cardiovascular effects were observed (24).

These results provide a rationale for large clinical trials to further initial attempts at fostering the beneficial metabolic role of HDL.

\section{Conclusion}

Extensive use of statins resulted in a substantial progress in combating CVD but there remains a residual metabolic risk. While statins reduce LDL levels, they are not fully adequate as mono- therapy to raise HDL or to correct HDL-associated CVD risk in subjects with low HDL. Statins only have a modest effect on HDL.

HDL is known to protect against atherosclerosis by promoting reverse cholesterol transport and also through antiinflammatory, antioxidative, antithrombotic and nitric oxide effects. Individuals with above average level of HDL have been known to have improved survival up to age 85 (25). Low serum levels of HDLC or of apolipoprotein A-1 (ApoA-1), the major protein of HDL particles, are consistently associated with increased risk for all forms of atherosclerotic disease and its clinical sequelae, including myocardial infarction, stroke, and sudden death.

Decisive progress in effectively using the favorable potential of HDL has been tempered by the complexity of the HDL mechanism of action and by limited ablity to measure individual stages of their function. HDL is a complex therapeutic target, albeit one with immense potential for the treatment of patients with atherosclerosis. The clinical utility of elevating high-density lipoprotein cholesterol (HDL-C) to reduce risk for cardiovascular morbidity and mortality remains the subject of much debate. At present, raising HDL level holds great promise, on the basis of epidemiology and initial trials, but we have to await the outcome of several large clinical trials currently under way to define the clinical role of older and novel therapies to raise HDL-C level (26).

\section{References}

1. Ginter E, Simko V. Statins: the drugs for the 21 st century? Bratisl Lek Listy 2009; 110 (10): 664-668.

2. European Health for All database (HFA-DB). Copenhagen, WHO Regional Office for Europe, [2010] http://euro.who.int/hfadb.

3. Steinberg D. Thematic review series: the pathogenesis of atherosclerosis: an interpretive history of the cholesterol controversy, part III: mechanistically defining the role of hyperlipidemia. J Lipid Res 2005; 46: 2037-2051.

4. Sharma RK, Singh VN, Reddy HK. Thinking beyond low-density lipoprotein cholesterol: strategies to further reduce cardiovascular risk. Vasc Health Risk Manag 2009; 5: 793-799.

5. Rocha VZ, Libby P. Obesity, inflammation, and atherosclerosis. Nature Rev Cardiol 2009; 6: 399-409.

6. Francis GA. The complexity of HDL. Biochim Biophys Acta 2010; 1801 (12): 1286-1293.

7. Nicholls SJ, Uno K, Tuzcu EM et al. Lessons from coronary intravascular ultrasound on the importance of raising high-density lipoprotein cholesterol. Curr Atheroscler Rep 2010; 12 (5): 301-307.

8. Schaefer EJ, Santos RD, Asztalos BF. Marked HDL deficiency and premature coronary heart disease. Curr Opin Lipidol 2010; 21 (4): 289-297.

9. Hirayama S, Miida T, Miyazaki O et al. Pre beta1-HDL concentration is a predictor of carotid atherosclerosis in type 2 diabetic patients. Diabetes Care 2007; 30 (5): 1289-1291.

10. Heinecke J. HDL and cardiovascular-disease risk--time for a new approach? N Engl J Med 2011; 364 (2): 170-171.

11. Khera AV, Cuchel M, de la Llera-Moya M et al. Cholesterol efflux capacity, high-density lipoprotein function, and atherosclerosis. N Engl J Med 2011; 364 (2): 127-135. 
$172-176$

12. Remaley AT, Warnick GR. The changing testing paradigm. Clin Lab News 2007; 33 (12).

13. Liu HR, Wu G, Zhou B et al. Low cholesteryl ester transfer protein and phospholipid transfer protein activities are the factors making three shrew and beijing duck resistant to atherosclerosis. Lipids Health Dis 2010; 9: 114 .

14. Toth PP, Davidson MH. High-density lipoproteins: marker of cardiovascular risk and therapeutic target. J Clin Lipidol 2010; 4 (5): 359-364.

15. Haas MJ, Mooradian AD. Inflammation, high-density lipoprotein and cardiovascular dysfunction. Curr Opin Infect Dis 2011; Feb 14.

16. Holzer M, Gauster M, Pfeifer T et al. Protein carbamylation renders high-density lipoprotein dysfunctional. Antioxid Redox Signal 2011; Jan 14.

17. Skaggs BJ, Hahn BH, Sahakian L et al. Dysfunctional, pro-inflammatory HDL directly upregulates monocyte PDGFR $\beta$, chemotaxis and TNF $\alpha$ production. Clin Immunol 2010; 137 (1): 147-156.

18. van Dujinhoven FJ, Bueno-de-Mesquito $\mathrm{HB}$, Calligaro $M$ et al. Blood lipid and lipoprotein concentrations and colorectal cancer risk in the European Prospective Investigation into cancer and nutrition.Gut 2011, March 7.

19. Burillo E, Andres EM, Mateo-Gallego R et al. High-density lipoprotein cholesterol increase and non-cardiovascular mortality: a meta-analysis. Heart 2010; 96 (17): 1345-1351.
20. Ragbir S, Farmer JA. Dysfunctional high-density lipoprotein and atherosclerosis. Curr Atheroscler Rep 2010; 12 (5): 343-348.

21. Duffy D, Rader DJ. Update on strategies to increase HDL quantity and function. Nat Rev Cardiol 2009; 6: 455-463.

22. Hausenloy DJ, Yellon DM. Targeting residual cardiovascular risk: raising high-density lipoprotein cholesterol levels. Heart 2008; 94 (6): 706-714.

23. Yvan-Charvet L, Kling J, Pagler T et al. Cholesterol efflux potential and antiinflammatory properties of high-density lipoprotein after treatment with niacin or anacetrapib. Arterioscler Thromb Vasc Biol 2010; 30 (7): 1430-1438.

24. Cannon CP, Shah S, Dansky HM et al. Safety of Anacetrapib in patients with or at high risk for coronary heart disease. N Engl J Med 2010; 363: 2406-2415.

25. Rahilly-Tierney CR, Spiro A, Vokonas $P$ et al. Relation between high-density lipoprotein cholesterol and survival to age 85 years in men (from the VA Normative Aging Study). Am J Cardiol 2011; 4.

26. Natarajan P, Ray KK, Cannon CP. High-density lipoprotein and coronary heart disease: current and future therapies. J Am Coll Cardiol 2010; 55 (13): 1283-1299.

Received March 30, 2011. Accepted December 17, 2012. 